\title{
Universiteit
}

Leiden

The Netherlands

\section{Critical and noncritical jamming of frictional grains}

Somfai, E.; Hecke, M.L. van; Ellenbroek, W.G.; Shundyak, K.; Saarloos, W. van

\section{Citation}

Somfai, E., Hecke, M. L. van, Ellenbroek, W. G., Shundyak, K., \& Saarloos, W. van. (2007). Critical and noncritical jamming of frictional grains. Physical Review E, 75(2), 020301. doi:10.1103/PhysRevE.75.020301

Version: $\quad$ Publisher's Version

License: $\quad$ Leiden University Non-exclusive license

Downloaded from: https://hdl.handle.net/1887/66536

Note: To cite this publication please use the final published version (if applicable). 


\title{
Critical and noncritical jamming of frictional grains
}

\author{
Ellák Somfai, ${ }^{1, *}$ Martin van Hecke, ${ }^{2}$ Wouter G. Ellenbroek, ${ }^{1}$ Kostya Shundyak, ${ }^{1}$ and Wim van Saarloos ${ }^{1}$ \\ ${ }^{1}$ Instituut-Lorentz, Universiteit Leiden, Postbus 9506, 2300 RA Leiden, The Netherlands \\ ${ }^{2}$ Kamerlingh Onnes Lab, Universiteit Leiden, Postbus 9504, 2300 RA Leiden, The Netherlands
}

(Received 19 October 2005; published 2 February 2007)

\begin{abstract}
We probe the nature of the jamming transition of frictional granular media by studying their vibrational properties as a function of the applied pressure $p$ and friction coefficient $\mu$. The density of vibrational states exhibits a crossover from a plateau at frequencies $\omega \gtrsim \omega^{*}(p, \mu)$ to a linear growth for $\omega \lesssim \omega^{*}(p, \mu)$. We show that $\omega^{*}$ is proportional to $\Delta z$, the excess number of contacts per grain relative to the minimally allowed, isostatic value. For zero and infinitely large friction, typical packings at the jamming threshold have $\Delta z \rightarrow 0$, and then exhibit critical scaling. We study the nature of the soft modes in these two limits, and find that the ratio of elastic moduli is governed by the distance from isostaticity.
\end{abstract}

DOI: 10.1103/PhysRevE.75.020301

PACS number(s): 45.70. $-\mathrm{n}, 46.65 .+\mathrm{g}$

Granular media, such as sand, are conglomerates of dissipative, athermal particles that interact through repulsive and frictional contact forces. When no external energy is supplied, these materials jam into a disordered configuration under the action of even a small confining pressure [1]. In recent years, much new insight has been amassed about the jamming transition of models of deformable, spherical, athermal, frictionless particles in the absence of gravity and shear [2]. The beauty of such systems is that they allow for a precise study of the jamming transition that occurs when the pressure $p$ approaches zero (or, geometrically, when the particle deformations vanish). At this jamming point $J$ and for large systems, the contact number [3] equals the so-called isostatic value $z_{\text {iso }}^{0}$ (see below), while the packing density $\phi_{\mathrm{J}}^{0}$ equals random close packing $[2,4]$. Moreover, for compressed systems away from the jamming point, the pressure $p$, the excess contact number $\Delta z=z(p)-z_{\text {iso }}^{0}$, and the excess density $\Delta \phi=\phi-\phi_{\mathrm{J}}^{0}$ are related by power-law scaling relations - any one of the parameters $p, \Delta z$, and $\Delta \phi$ is sufficient to characterize the distance to jamming.

Isostatic solids are marginal solids-as soon as contacts are broken, extended "floppy modes" come into play [5]. Approaching this marginal limit in frictionless packings as $p \rightarrow 0$, the density of vibrational states (DOS) at low frequencies is strongly enhanced-the DOS has been shown to become essentially constant up to some low-frequency crossover scale $\omega^{*}$, below which the continuum scaling $\sim \omega^{d-1}$ is recovered $[2,6-11]$. For small pressures, $\omega^{*}$ vanishes $\sim \Delta z$. This signals the occurrence of a critical length scale, when translated into a length via the speed of sound, below which the material deviates from a bulk solid [9]. The jamming transition for frictionless packings thus resembles a critical transition.

In this paper we address the question whether an analogous critical scenario occurs near the jamming transition at $p=0$ of frictional packings. The Coulomb friction law states that, when two grains are pressed together with a normal force $F^{\mathrm{n}}$, the contact can support any tangential friction force

\footnotetext{
*Present address: Department of Physics, Oxford University, 1 Keble Roard, Oxford OX1 3NP, United Kingdom.
}

$F^{\mathrm{t}}$ with $F^{\mathrm{t}} \leq \mu F^{\mathrm{n}}$, where $\mu$ is the friction coefficient. In typical packings, essentially none of these tangential forces is at the Coulomb threshold $F^{\mathrm{t}}=\mu F^{\mathrm{n}}[8,12]$. A crucial feature of these packings of frictional particles is that, for $p \rightarrow 0$, they span a range of packing densities and have a nonunique contact number $z_{\mathrm{J}}(\mu)$, which typically is larger than the frictional isostatic value $z_{\text {iso }}^{\mu}=d+1$ [12-15] (see below). So two questions arise. Do frictional systems ever experience a "critical" jamming transition in the sense that $\omega^{*}$ vanishes when $p \rightarrow 0$ ? What is the nature of the relations between $p$, $\mu, \omega^{*}$, excess contact number $z(\mu, p)-z_{\mathrm{J}}(\mu)$, and excess density?

The results that we present below give convincing evidence that jamming of frictional grains should be seen as a two-step process. The first step is the selection of $z$ for fixed $\mu$ and a given numerical procedure [see Fig. 1(a)]; this has been studied before [12-15]. Our focus here is on the second step, the fact that the critical frequency $\omega^{*}$ of the DOS of vibrations of infinitesimal amplitude is proportional to the distance to the frictional isostatic point $\Delta z:=z(\mu, p)-z_{\text {iso }}^{\mu}$. The crucial point is that $z_{\mathrm{J}}(\mu)$ and $z_{\text {iso }}^{\mu}$ in general differ. In particular, for small values of $\mu$, the contact number saturates at a value substantially above the isostatic limit, $\omega^{*}$ saturates at a finite value and the system remains far from criticality. For increasing values of $\mu$, however, $z_{\mathrm{J}}(\mu)$ approaches $z_{\text {iso }}^{\mu}$, and thus for large friction values $\omega^{*}$ evidences an increasingly large scale near the jamming point. The vanishing of $\omega^{*}$ has its origin in the emergence of floppy modes at the isostatic point. We show that, as in frictionless systems, both $\omega^{*}$ and the ratio of shear to compression modulus, $G / K$, scale as $\Delta z$. In short, the distance to isostaticity, which is well defined, governs the scaling of both frictional and frictionless systems, providing a unified picture of jamming of weakly compressible particles.

Let us, before presenting our results, recapitulate the wellknown counting arguments for the contact number in the limit $p \rightarrow 0$ for dimension $d$ [4]. Since the deformation of the spheres vanishes in the limit $p=0$, all particles in contact are at a prescribed distance, which gives $z N / 2$ constraints on the $d N$ particle coordinates, leading to $z \leq 2 d$. For the frictionless case, the $z N / 2$ normal contact forces are constrained by $N d$ force balance equations-hence only for $z \geq 2 d$ can we ge- 

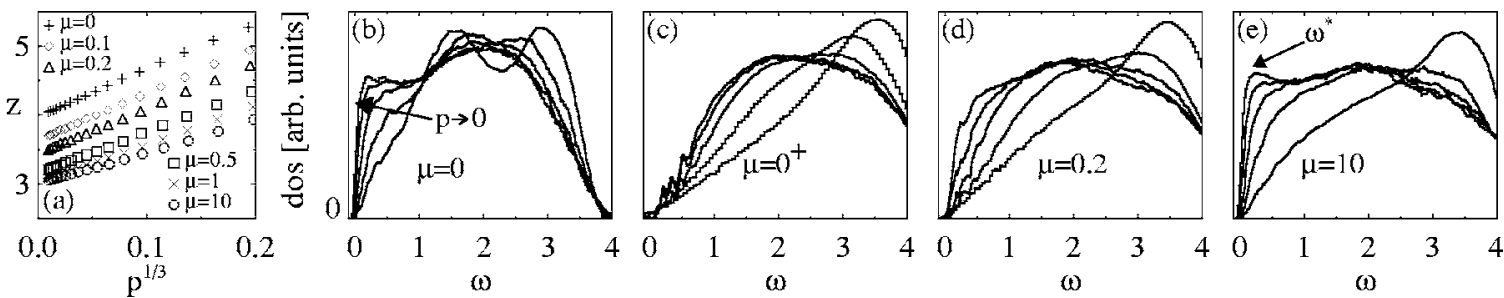

FIG. 1. (a) Average contact number $z$ as a function of $p^{1 / 3}$ for various $\mu$ as indicated. (b)-(e) Vibrational DOS for granular packings for friction coefficients as indicated, and for pressures approximately $5 \times 10^{-6}, 5 \times 10^{-5}, 5 \times 10^{-4}, 4 \times 10^{-3}$, and $3 \times 10^{-2}$. For decreasing $p$ the DOS becomes steeper for small $\omega$, and the crossover frequency $\omega^{*}$, indicated in (e), decreases with $p$. The packing with $\mu=0^{+}$is obtained by first making a frictionless packing and then turning on the tangential frictional forces in the DOS calculation. As noted in the text, all frequencies are scaled by a factor $p^{1 / 6}$.

nerically find a set of balancing forces [16]. Taken together, this yields $z \rightarrow 2 d=: z_{\text {iso }}^{0}$ as $p \rightarrow 0$ : at the jamming transition, packings of frictionless spheres are isostatic. For frictional packings, there are $z d N / 2$ contact force components constrained by $d N$ force and $d(d-1) N / 2$ torque balance equations - thus $z \geq d+1$, with $z_{\text {iso }}^{\mu}=d+1$ the isostatic value. Hence, at the jamming transition, frictional spheres do not have to become isostatic but can attain contact numbers between $z_{\text {iso }}^{\mu}=d+1$ and $2 d$. While it is not well understood what selects the contact number of a frictional packing at $J$, simulations for disks in two dimensions show that in practice $z_{\mathrm{J}}(\mu)$ is a decreasing function of $\mu$, ranging from 4 at small $\mu$ to 3 for large $\mu$ [12-14]; see also Fig. 1(a).

Procedure. Our numerical systems are two-dimensional (2D) packings of 1000 polydisperse spheres that interact through 3D Hertz-Mindlin forces [17], contained in square boxes with periodic boundary conditions. We set the Young modulus of the spheres $E^{*}=1$, which becomes the pressure unit, and set the Poisson ratio to zero. Our unit of length is the average grain diameter, the unit of mass is set by asserting that the grain material has unit density, and the unit of time follows from the speed of sound of pressure waves inside the grains [10]. The packings are constructed by cooling while slowly inflating the particle radii in the presence of a linear damping force, until the required pressure is obtained. For each value of $\mu$ and $p, 20$ realizations are constructed (occasional runs with 100 realizations did not improve accuracy).

Once a packing is made, the additional damping force is switched off and the dynamical matrix is obtained by linearizing the equations for small-amplitude motions, which include both rotations and translations. It is important to realize the special role of the friction: if the density of contacts that precisely satisfy $F^{\mathrm{t}}=\mu F^{\mathrm{n}}$ is negligible, the Coulomb condition $F^{\mathrm{t}} \leq \mu F^{\mathrm{n}}$ only plays a crucial role during the preparation of a packing. We will assume that this is the case, and come back to this subtle point later. Under these assumptions, and for arbitrarily small-amplitude vibrations, the Coulomb condition is automatically obeyed and the value of $\mu$ no longer plays a role in analyzing the vibrational modes. Moreover, the changes in $F^{\mathrm{t}}$ are then nondissipative and the eigenmodes of the dynamical matrix are undamped. In this picture, the main role of the value of the friction coefficient is in tuning the contact number.

We analyze the density of vibrational states of the pack- ings thus obtained. Since for Hertzian forces the effective spring constants scale with the overlap $\delta$ as $d F^{\mathrm{n}} / d \delta \sim \delta^{1 / 2}$ $\sim p^{1 / 3}$ [17], all frequencies will have a trivial $p^{1 / 6}$ dependence. To facilitate comparison with data on frictionless spheres with one-sided harmonic springs $[2,8]$, we report our results in terms of scaled frequencies in which this $p^{1 / 6} \mathrm{de}$ pendence has been taken out.

Variation of $z$. Anticipating the crucial role of the contact number, we start by presenting $z(\mu, p)$ for our packings. Figure 1(a) confirms the earlier observations [12-14] that the effective value of $z_{\mathrm{J}}(\mu) \equiv z(\mu, p \rightarrow 0)$ varies from about 4 to about 3 when $\mu$ is increased. Moreover, the excess number of contacts $z(\mu, p)-z_{\mathrm{J}}(\mu)$ varies with pressure as $p^{1 / 3}$ for all values of $\mu$.

DOS. Figures 1(b)-1(e) show our results for the DOS for various values of $\mu$. For the frictionless case shown in Fig. $1(\mathrm{~b})$, we recover the gradual development of a plateau in the density of states as the pressure is decreased [2,8]. For this case $z \rightarrow z_{\text {iso }}^{0}$, and the crossover frequency $\omega^{*}$ scales as $\Delta z$ $=z-z_{\text {iso }}^{0}[7-9]$ (see below). However, as Figs. 1(c) and 1(d) illustrate, as soon as the tangential frictional forces are turned on, this enhancement of the DOS at low frequencies largely disappears, because the frictionless floppy modes are destroyed. This point is demonstrated most dramatically in Fig. 1(c), where the underlying packing has been generated for zero friction, and the friction is only switched on when calculating the DOS - this represents the limit of vanishingly small but nonzero friction, for which the DOS is seen to be very far from critical. By increasing the friction coefficient, the development of a plateau and the scaling of the crossover progressively reappear [Fig. 1(e)]. The intuitive picture that emerges is that, with increasing friction, granulates at the jamming point approach criticality.

In order to back this up quantitatively, we perform a scaling analysis of the low-frequency behavior of these DOS $\left(D_{S}\right)$. To avoid binning problems, we work with the integrated density of states $I(\omega)=\int{ }^{\omega} d \omega^{\prime} D_{S}\left(\omega^{\prime}\right)$. The critical frequencies are then obtained by requiring that the rescaled integrated DOS, $\left(\omega^{*}\right)^{-1} I\left(\omega / \omega^{*}\right)$ collapse. Such collapse is never perfect, in particular since not all DOS have precisely the same "shape" (Fig. 1). We vary the value of $\omega_{\text {overlap }}$ $:=\omega / \omega^{*}$ where we require the rescaled integrated DOS to overlap-as Fig. 2(a) illustrates, this yields precise values for $\omega^{*}$ as function of $\omega_{\text {overlap. Restricting ourselves to the cross- }}$ over regime $\left(1<\omega_{\text {overlap }}<3\right)$, we obtain by this procedure 

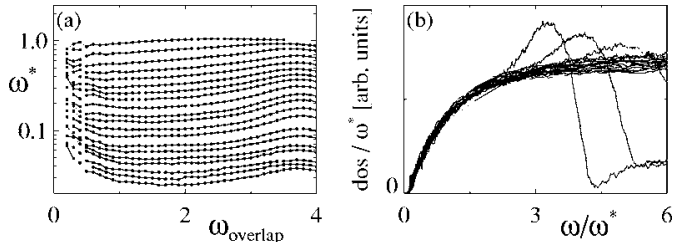

FIG. 2. (a) $\omega^{*}$ for $\mu=10$ as a function of $\omega_{\text {overlap }}$ the regime $\left(1<\omega_{\text {overlap }}<3\right)$ corresponds to the crossover regime in the DOS that we focus on here [see text and (b)]. (b) The rescaled DOS for $\mu=10$ exhibit good data collapse in the crossover regime. Here 20 rescaled DOS are shown with $p$ ranging from $9 \times 10^{-7}$ to $3 \times 10^{-2}$.

both an estimate of $\omega^{*}$ and of its error bar. As Fig. 2(b) illustrates, when rescaled with these estimated values of $\omega^{*}$, the collapse of the DOS in the crossover regime is convincing.

Scaling of $\omega^{*}$. The first main result of this paper is shown in Fig. 3: $\omega^{*}$ does not scale in a simple way with $p$, but the data for all $\mu$ and $p$ collapse onto a single curve when plotted as a function of $\Delta z=z-z_{\text {iso }}^{\mu}$ [Figs. 3(a) and 3(c)]. Moreover, $\omega^{*} \sim \Delta z$ - the plot of $z-3$ versus $p$ shown in Fig. 3(b) is essentially equivalent to the plot of $\omega^{*}$ vs $p$. In other words, packings with $\Delta z \ll 1$ have many low-lying vibration modes and correspondingly a large enhancement (plateau) in the DOS. The dominant quantity governing the behavior of frictional granular media is the distance from the frictional "critical point" $z=z_{\text {iso }}^{\mu}=d+1=3$. This distance can be characterized most conveniently by $\Delta z$-put in these terms, the scaling of $\omega^{*}$ for frictional media is very similar to the scaling for frictionless media shown in Figs. 3(d) and 3(e).

Scaling of elastic moduli. The contact number for isostatic systems reaches the minimum needed to remain stablehence additional broken bonds then generate global zeroenergy displacement modes, so-called floppy modes $[2,5]$. For frictionless systems, the excess of soft modes and development of a plateau in the DOS for small $\Delta z$ are intimately connected to these floppy modes $[7,9,18]$. For frictionless systems they also cause the shear modulus $G$ to become
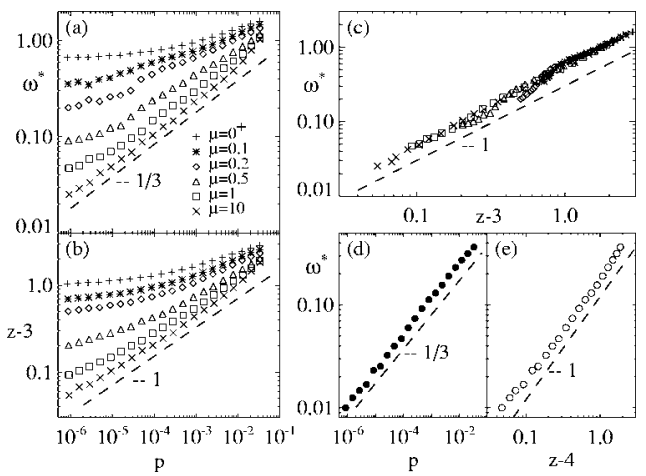

FIG. 3. (a) $\omega^{*}$ as a function of pressure $p$ for a range of friction coefficients $\mu$-error bars are similar to or smaller than symbol sizes. (b) Deviation from isostaticity for the same range of parameters. (c) $\omega^{*}$ scales linearly with the distance to isostaticity for frictional packings. (d), (e) $\omega^{*}$ for frictionless packings scales with both $p$ and $z-4$. Dashed lines indicate power laws with exponents as indicated. For details, see text.
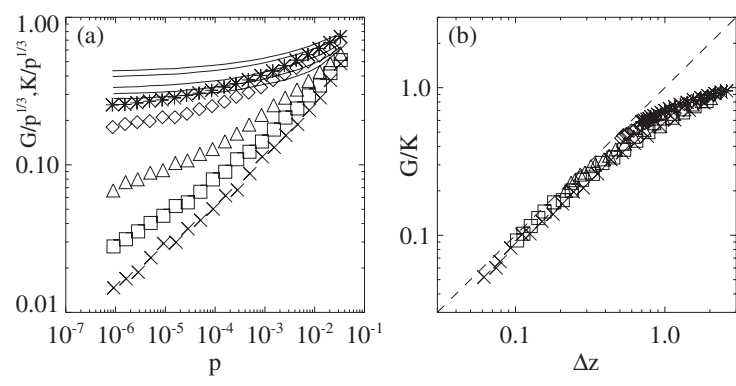

FIG. 4. Scaling of bulk modulus $K$ and shear modulus $G$ as function of $p$ and $\mu$-as for the data for $\omega^{*}$, the trivial $p^{1 / 3}$ dependence has been divided out. (a) The rescaled bulk modulus $K$ (curve) essentially levels off for small $p$, while the shear modulus $G$ (symbols as in Fig. 3) varies strongly with both $p$ and $\mu$. (b) $G / K$ scales like the excess contact number for small $\Delta z$.

much smaller than the bulk modulus $K$-in fact, $G / K \sim \Delta z$ $[2,11,18]$.

Our second main result is that we have found numerically that for frictional systems the ratio $G / K$ depends only on $\Delta z$ (and not on, e.g., $\mu$ ), and for small $\Delta z$ it also scales as $\Delta z$. To calculate the moduli, we start from the dynamical matrix which relates forces and displacements. Calculating, in linear order, the global stress resulting from an imposed deformation, the bulk and shear moduli are deduced [18]. Since for Hertzian forces the effective spring constants scale as $p^{1 / 3}$, we have divided out this trivial pressure dependence. The results of our calculations are shown in Fig. 4. As could be expected, the (rescaled) bulk modulus $K$ remains essentially constant. Surprisingly, the shear modulus $G$ becomes much smaller than $K$ for small $p$ and large $\mu$, and when plotted as a function of $\Delta z$, the ratio $G / K$ is found to scale as $\Delta z$, as was predicted in [11]. Hence, in packings of deformable spheres, both $\omega^{*}$ and $G / K$ scale with $\Delta z$, regardless of the presence of friction.

Discussion. The sudden change in the DOS when increasing $\mu$ from zero hints at the singular nature of the $\mu \rightarrow 0$ limit. On the one hand, the nature of the dynamical matrix suddenly changes in this limit because the rotational degrees of freedom which are irrelevant for $\mu=0$ turn on as soon as $\mu \neq 0$. On the other hand, we have recently found that, the more slowly the packings are allowed to equilibrate during their preparation, the more the density of fully mobilized contacts, i.e., those for which $F^{\mathrm{t}}=\mu F^{\mathrm{n}}$, tends to increase; in fact especially for small $\mu$ the fraction of fully mobilized contacts in slowly equilibrated samples becomes very substantial $[19,20]$. The effect of these fully mobilized contacts on the DOS depends on additional physical assumptions. For example, if we assume that, for some reason, the contacts remain constrained at the Coulomb threshold in the vibrational dynamics, we expect them to have an enhanced DOS for small pressures at all $\mu$. More likely, these contacts would slip, leading to an initial strongly nonlinear response after which no contacts would be fully mobilized any more, and our results for the DOS would go through essentially unchanged.

Note that even a small difference between dynamic and static friction could suppress the effect of the fully mobilized contacts. Moreover, for realistic values of the friction $(\mu$ 
$\gtrsim 0.7$, say) these effects are not very important since there the fraction of fully mobilized contacts is small. Thus, the results of this paper will apply directly to packings with experimentally relevant values of the friction.

A second issue that deserves further attention is the nature of the soft modes. Our scaling result for $G / K$ suggests that for frictional systems these are dominated by shearlike (volume-conserving) deformations, just as for frictionless systems. Apparently, rotations and particle motions couple such as to allow large-scale floppy-mode-like distortions of frictional isostatic packings. Indeed, numerically obtained low-frequency eigenmodes of frictional and frictionless systems look remarkably similar. Whether the scaling of $\omega^{*}$, the scaling of $G / K$, and the nature of floppy modes are similarly related in more general systems, such as packings of frictionless or frictional nonspherical particles, is an important question.

Outlook. Our study of the density of vibrational states for frictional systems gives strong evidence for a scenario partly analogous to the one for frictionless packings: frictional granular media become critical and exhibit scaling when their contact number approaches the isostatic limit. But there is an important difference from the frictionless case: while there the isostatic limit is automatically reached in the hard-particle-small- $p$ limit, this is not necessarily so for the frictional case-here $p$ and $z$ are not directly related, and only for large friction does $z$ approach isostaticity at small pressures. This isostatic point is relevant in practice: most materials have a value of $\mu$ of order 1, and as Figs. 3 and 4 illustrate, one then observes approximate scaling over quite some range.

We are grateful to M. Depken, L. Silbert, S. Nagel, D. Frenkel, H. van der Vorst, and T. Witten for illuminating discussions. E.S. acknowledges support from the EU network PHYNECS, W.E. support from the physics foundation FOM, and M.v.H. support from NWO/VIDI.
[1] A. J. Liu and S. Nagel, Nature (London) 396, 21 (1998).

[2] C. S. O’Hern et al., Phys. Rev. E 68, 011306 (2003); Phys. Rev. Lett. 88, 075507 (2002).

[3] We will use the convention that the superscript distinguishes frictionless (0) from frictional $(\mu)$ quantities, while the subscript indicates whether quantities are taken at the jamming $(J)$ or isostatic (iso) point.

[4] C. F. Moukarzel, Phys. Rev. Lett. 81, 1634 (1998).

[5] S. Alexander, Phys. Rep. 296, 65 (1998).

[6] A. Tanguy et al., Phys. Rev. B 66, 174205 (2002).

[7] M. Wyart, S. R. Nagel, and T. A. Witten, Europhys. Lett. 72, 486 (2005).

[8] L. E. Silbert, A. J. Liu, and S. R. Nagel, Phys. Rev. Lett. 95, 098301 (2005); L. E. Silbert et al., Phys. Rev. E 65, 031304 (2002).

[9] M. Wyart et al., Phys. Rev. E 72, 051306 (2005).

[10] E. Somfai et al., Phys. Rev. E 72, 021301 (2005).

[11] M. Wyart, Ann. Phys. (Paris) 30(3), 1 (2005).

[12] A. Kasahara and H. Nakanishi, Phys. Rev. E 70, 051309
(2004).

[13] T. Unger, J. Kertész, and D. E. Wolf, Phys. Rev. Lett. 94, 178001 (2005)

[14] H. P. Zhang and H. A. Makse, Phys. Rev. E 72, 011301 (2005); H. A. Makse et al., ibid. 70, 061302 (2004).

[15] G. Y. Onoda and E. G. Liniger, Phys. Rev. Lett. 64, 2727 (1990).

[16] In the counting argument, $N$ refers to the number of nonrattling particles.

[17] That is normal force $F^{\mathrm{n}} \sim \delta^{3 / 2}$ with $\delta$ the overlap between particles, tangential force increment $d F^{\mathrm{t}} \sim \delta^{1 / 2} d t$ with $d t$ the relative tangential displacement change, provided $F^{\mathrm{t}} \leq \mu F^{\mathrm{n}}$.

[18] W. G. Ellenbroek et al., Phys. Rev. Lett. 97, 258001 (2006).

[19] K. Shundyak et al., e-print cond-mat/0610205.

[20] When fully mobilized contacts are considered to be fixed at the Coulomb threshold, gently prepared packings are found to approach a generalized isostaticity line at small pressures for any $\mu$ [19], while less gently prepared packings will have less fully mobilized contacts $[8,12]$. 